\title{
The Effectiveness of Online Stress Management Training Interventions: A Systematic Literature Review
}

\author{
Dr. Jessica van Wingerden MBA MCC (Corresponding author) \\ Schouten Global, Centre of Knowledge \& Innovation \\ P.O. Box 266, 5300 AG Zaltbommel, The Netherlands \\ E-mail: jessica.vwingerden@sn.nl; jessica.vanwingerden@gmail.com \\ Dr. Daantje Derks \\ Erasmus University Rotterdam \\ The Netherlands
}

Received: July 16, 2018 Accepted: August 4, 2018 Published: September 3, 2018

doi:10.5296/ijld.v8i3.13600 URL: https://doi.org/10.5296/ijld.v8i3.13600

\begin{abstract}
The central aim of this systematic literature review study was to investigate the effectiveness of online stress management training interventions that aimed to improve employees' well-being. The study focused both on the effectiveness of online stress management training interventions and the sustainability of the intervention effects over time. Within this literature review 18 intervention studies, conducted worldwide among 3085 participants between 2002 and 2017, were evaluated. Methodological quality was examined using the Mixed Methods Appraisal Tool (MATT). In general, the main outcomes showed that most of the interventions turned out to be effective in decreasing employees' levels of stress. In addition, some of these studies also revealed sustainability of intervention effects over time. This suggests that online stress management interventions are a promising tool for organizations to foster employee well-being.
\end{abstract}

Keywords: Systematic literature review, online training interventions, stress management, employee well-being, sustainability of intervention effects

\section{Introduction}

Work related stress has a big impact on contemporary organizations in Western Society. The costs of work related stress are found to be over hundreds of billions annually in Europe, 
America and the UK (EU-OSHA, 2010; Riedell, Lynch, Baase, Hymel, \& Peterson, 2010). These costs are related to decreased productivity as a result of health problems caused by work related stress, missed work days and health care costs. Therefore, organizations are willing to invest time and money in training interventions that aim to reduce work related stress and enhance employee well-being (Aldana, 2001). Over the last fifteen years, online training interventions aimed at enhancing well-being and reducing work related stress were offered to employees in organizations around the world. An important research question is whether these online interventions are indeed effective in enhancing employee well-being and to what extent these potential intervention effects are sustainable over time. This paper explores these questions by reviewing the literature on online stress management interventions among organizations in different sectors all over the world. An overview of online stress management interventions, including training frequency, training hours, effectiveness and sustainability are provided. As such, this study adds to the learning and development literature by providing a state-of-the-art overview of the impact of online stress management training interventions on employee well-being. Subsequently, the results of the literature review and implications are discussed.

\section{Theory}

\subsection{The Impact of Work Related Stress on Employee' Well-Being}

Work related stress is a global issue and according to several studies the number one occupational disease in Western society. The Health and Safety Executive (Aldana, 2001). showed that work related stress in the UK costs about 3.7 billion pound every year. In addition, the report revealed that five million employees feel "very" or "extremely" stressed by their work, and half a million employees experience work-related stress at a level that they believe it is making them ill. In line with these findings, a study by Randolfi (1997), revealed that work related stress caused health problems that led to decreased productivity in organizations. Companies in America spend about 300 billion dollar annually for health care and missed work days as a result of workplace-related stress (Eastern Kentucky University, 2016). In addition, around, $40 \%$ of American workers say that their jobs are very or extremely stressful and at the same time, $26 \%$ of employees say they are very often burned out, or stressed at the workplace. In a similar vein, the European Survey of Enterprises on New and Emerging Risk (2010). revealed that work related stress is a risk for our society, both in terms of costs and in terms of employee' well-being. Moreover, the ESENER report revealed that $79 \%$ of European managers are concerned about stress in their workplaces and recognize interventions are needed to solve this complex issue. In line with this sense of urgency by managers, organizations are willing to invest time and money in organizational interventions aimed at improving employee well-being (Aldana, 2001). Over te last decades, organizational interventions aimed at improving employee well-being were implemented in practice and turned out to have potential to reduce work related stress and increase employee' well-being (Giga, Noblet, Faragher \& Cooper, 2003; Meyers, Van Woerkom, \& Bakker, 2013; Van Wingerden, Bakker, \& Derks, 2017). Since technological advantages have made the internet an essential part of today's business, contemporary employers are offering their employees online interventions to enhance their well-being (Cook, Billings, Hersch, Back, \& Hendrickson, 2007; 
Shimazu, Kawakami, Irimajiri, Sakamoto, \& Amano, 2005).

\subsection{Online Training Interventions for Employee Well-Being}

The Internet plays an important role in employees' learning and development processes, and the prevalence of online learning in the workplace is still growing (Schlager, Fusco, \& Schank, 2002). Online interventions in the workplace offer several advantages for both employees and organizations. In comparison to face-to-face interventions, online interventions do have advantages in relation to efficiency, scalability and flexibility (Stralen, de Vries, Mudde, Bolman, \& Lechner, 2011). These types of interventions are cost-effective, have the ability to reach large numbers of individuals and can be easily tailored to individual needs (Napolitano et al., 2003). Because of these advantages, there is not only a growing interest in using online interventions for learning and development in the workplace, but also to enhance well-being at work (Cook et al., 2007, Shimazu et al., 2005). As underlined earlier by Mitchell, Vella-Brodrick and Klein (2010), there is still a small body of research on online interventions for well-being. In a review paper by Mitchell et al. (2010), five different online interventions were analyzed. The overview of characteristics reported in this study revealed that three out of the five intervention studies showed an increase of participants' well-being. However, only one intervention study contained a sample of employees, the other intervention studies in the review consisted of samples of adults with mild depression. The intervention study that aimed to enhance employee's resilience did not reveal any significant effects. In contrast, a literature review of fifteen articles on different positive psychology interventions in organizations by Meyers, Woerkom and Bakker (2013), contained fourteen classroom interventions and one online intervention. The online intervention study revealed a significant effect on participants' well-being. However, this study did not use a follow up measurement after the post-test, so we do not know whether the initial positive effects on employee well-being were sustainable over time. In line with this observation, more research on online training interventions for employee well-being is needed to get a better understanding of the full potential of these types of interventions. Over the last decade, multiple well-being interventions that already proofed their benefits and potential in a clinical or therapeutically setting, found their way to the workplace (Giga et al., 2003; Avey, Wernsing, \& Luthans, 2008; Le Fevre, Kolt, \& Matheny, 2006; Thiart, Lehr, Ebert, Berking, \& Riper, 2015). One type of these interventions that aim to enhance employee well-being, are online stress management training interventions. This literature review will focus on the effectiveness of these online stress management training interventions for employee well-being and the sustainability of the initially found intervention effects.

\section{Method: Search Strategy, Quality Assessment and Analysis Strategy}

\subsection{Search Strategy}

A systematic literature search was conducted using three databases: Scopus, Web of Science and PsychINFO. The literature was searched using the search terms deduced from the research question, which are summarized in Table 1. In order to limit the amount of results and increase the relative amount of relevant results, rows 1 and 2 were limited to searching by title only. In addition, in order to maintain the actuality of this review, we only included articles that were published in English between 2002 and 2017. The initial search yielded a total of 64 findings, 


\section{Macrothink}

International Journal of Learning and Development

ISSN 2164-4063 2018, Vol. 8, No. 3

after removal of duplicates, 29 findings remained for further quality assessment (see Figure 1).

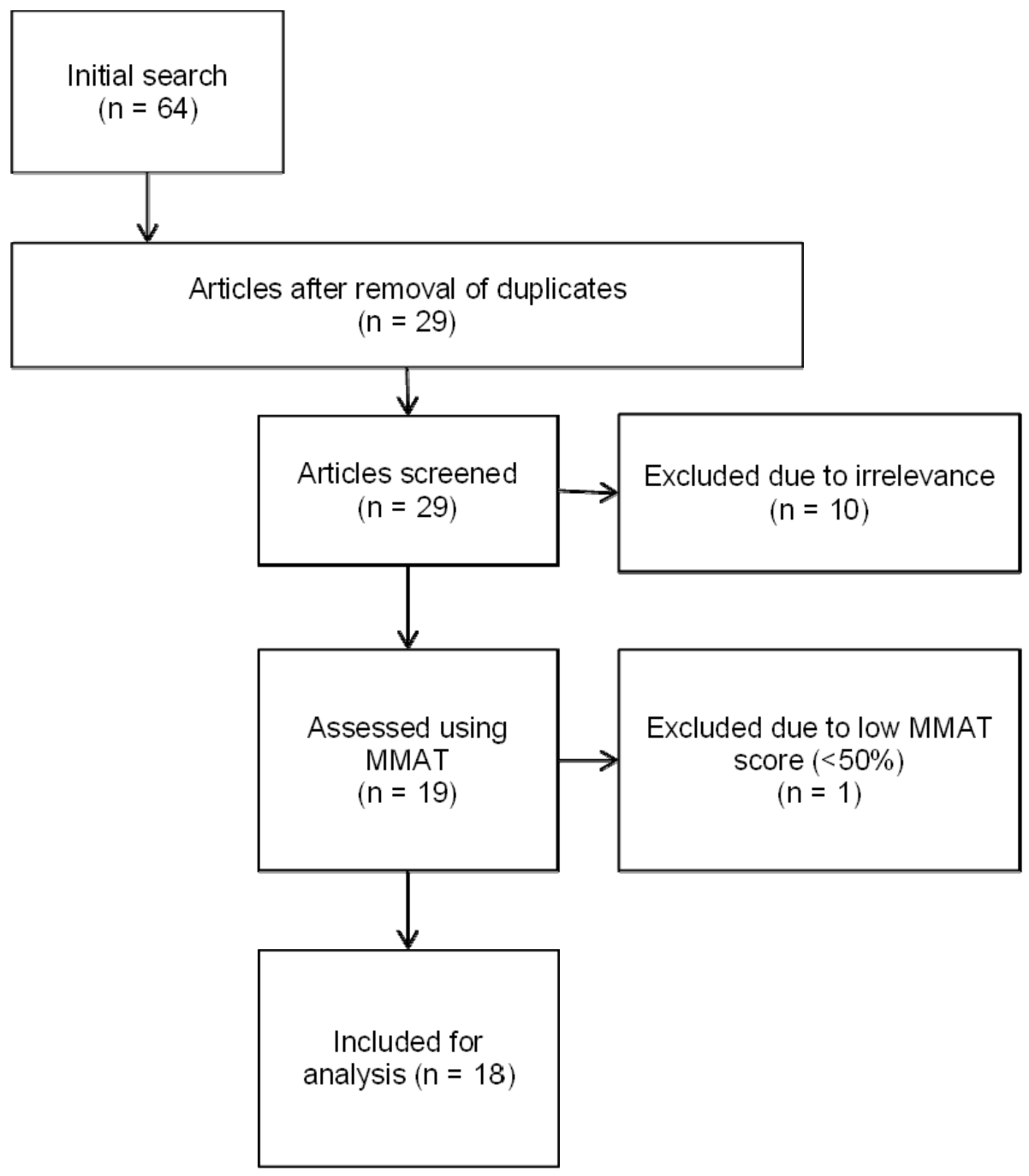

Figure 1. Flowchart Summarizing the Various Stages of Literature Search and Selection 


\section{I Macrothink}

Table 1. Summary of the Search Terms Used in Scopus, Web of Science and PsychINFO to Provide the Initial Literature Search

\begin{tabular}{|c|c|c|c|}
\hline Row & & Search terms & Search restrictions ${ }^{1}$ \\
\hline 1 & AND & $\begin{array}{l}\text { Stress AND (manag* OR reduc* OR lower* OR decreas* OR diminish* } \\
\text { OR lessen OR weaken OR coping OR cope OR regulate OR handl* OR } \\
\text { deal }^{*} \text { ) }\end{array}$ & Title \\
\hline 2 & AND & $\begin{array}{l}\text { (web OR online OR internet OR digital) AND (Intervention OR } \\
\text { treatment OR therapy OR program OR training OR trial OR RCT) }\end{array}$ & Title \\
\hline 3 & AND & $\begin{array}{l}\text { organisation OR organization OR business OR company OR work OR } \\
\text { workplace OR "work floor" OR employee OR worker OR job }\end{array}$ & $\begin{array}{l}\text { Title/Abstract/Keyword } \\
\text { (Scopus); Keyword } \\
\text { (PsychINFO); Topic } \\
\text { (Web of Science) }\end{array}$ \\
\hline
\end{tabular}

Note. ${ }^{1}$ All searches were limited to findings published between 2002 and 2017

\subsection{Quality Assessment.}

To determine if the methodological quality of the remaining studies was sufficient, we applied the principles of the Mixed Methods Appraisal Tool (MMAT) (Pluye et al., 2011). Through means of a checklist, the MMAT is used to determine the quality of quantitative, qualitative and mixed methods studies (Pluye, \& Hong, 2014). The ability to appraise mixed methods studies makes MMAT stand out among other tools, and it is known to be a reliable tool to efficiently determine which studies are suited for systematic literature search (Pace et al., 2012). Thus, we used the principles defined by MMAT to assess the quality of our initial findings.

To begin quality assessment of the remaining 29 studies, we first determined the relevance of the studies' research questions and the degree to which the questions were answered by looking at the titles and abstracts. Irrelevant studies or studies that did not have retraceable full versions were excluded from further analysis. As such, 10 studies were excluded after the first screening, leaving 19 studies for further quality assessment. By answering the MMAT questions for methodological quality assessment, we were able to determine the quality of the remaining 19 studies (Pluye et al., 2011). Studies that had a low MMAT score $(<50 \%)$ were subsequently excluded, removing another study from further analysis. Thus, a total of 18 studies remained for the final analysis (see Figure 1).

\subsection{Analysis Strategy}

In order to compare findings, and thus answer our research questions, we created an overview consisting of sample characteristics, intervention types, control conditions, assessment, measures, outcomes and other notable characteristics for the 18 remaining studies (see Table 2). Using this overview, we identified the main themes within the remaining literature by looking for similar findings and clustering them accordingly.

\section{Results}

The search resulted in 18 articles with a total number of 3085 participants (sample sizes 
ranging from $N=30$ to $N=309$ ), living in the United States, Canada, Australia, Japan, China, the United Kingdom and different countries in Europe. Research has been conducted in a variety of organizations, operating in the field of healthcare, education, industry, construction, finance and assurance, IT, manufacturing, and government. An overview of the 18 studies and the characteristics of the online stress management interventions studies can be found in Table 2 .

\subsection{Effectiveness of Online Stress Management Training Interventions}

The eighteen online stress management interventions presented in Table 2, could be divided in five different categories. Namely; Cognitive- behavioral based stress management interventions, Psycho-education based stress management interventions, Assertion training to manage stress levels, Career identity stress management training and Self-help based stress management interventions. The eight cognitive-behavioral based stress management interventions, focused on problem solving, emotion regulation and communication (Ruwaard, Lange, Bouwman, Broeksteeg, \& Schrieken, 2007; Umanodan, Shimazu, Minami, \& Kawakami, 2014; Ebert et al., 2016a; Ebert et al., 2016b; Heber, Lehr, Ebert, Berking, \& Riper, 2016; Eisen, Allen, Bollash, \& Pescatello, 2008; Hasson, Anderberg, Tehorell, \& Arnetz, 2005; Billings, Cook, Hendrickson, \& Dove, 2008). These eight studies all measured the effect of the intervention on (perceived) stress. All interventions had a significant negative effect on (perceived) stress, except the intervention study of Umanodan et al. (2014), which did not reveal a significant decrease of (perceived) stress, nor a significant effect on other study variables. The effects of two psycho-education based stress management interventions were tested by Ly, Asplund and Andersson (2014) and Shimazu, Anderberg, Theorell and Arnetz (2005). The interventions in these two studies, focused on problem solving, communication and self-efficacy of employees. The studies both measured the effect on (perceived) stress, where the intervention study by Ly et al. (2014) revealed a significant decrease of stress, the intervention study by Shimazu et al. (2005) did not reveal a significant effect, nor on (perceived) stress or on the other study variables.

Yamagishi, Kobayashi, Makiko, Nagami, Shimazu and Kageyama (2007) used an assertion training to manage the stress levels, behaviors and attitudes of employees, while Yamagishi, Kobayashi and Nakamura (2008) focused on career identity stress management training. Yamagishi et al. (2007) showed that the assertion knowledge and behavior increased significantly and that (perceived) stress decreased significantly. Within the study of Yamagishi et al. (2008), career identity knowledge increased significantly. However, there were no significant changes in mental health and (perceived) stress. Further, there are three self-help based stress management interventions, conducted by: Van Straten, Cuijpers and Smits (2008), Villani, Cognetta, Toniolo, Grassi, Cipresso and Riva (2013) and Zetterqvist, Maanmies, Strom, \& Andersson (2003). The self-help stress management interventions mainly focused on relaxation, problem solving and time management skills to manage the stress levels of employees. The studies by Villani et al., (2013) and Zetterqvist et al., (2003) showed that coping skills increased significantly and (perceived) stress decreased significantly. The study by Van Straten et.al. (2008), revealed a significant decrease of depression and anxiety, but no effect on work related stress. Finally Jung et al., (2016) and Wolever et al., (2012) examined a 
Mind-body stress management intervention. The intervention by Jung et al. (2016) turned out to be effective in decreasing (perceived) stress and negative affect and in increasing coping techniques, resilience and emotional intelligence. The intervention by Wolever et al., (2012) turned out to be effective in decreasing stress and increasing mindfulness. Sixteen of the eighteen online stress management intervention studies, showed significant effects on the majority of the intervention variables, in contrast, two studies (Shimazu et al., 2005; Umanodan et al., 2014) showed no significant effects. Nine of the eighteen studies reported significant estimates of effect sizes, eight of these nine studies reported Cohen's $d$. Further, four of the eighteen studies distinguished between 'within group' and 'between group' effect sizes, where the effect sizes for the experimental groups varied widely $(-0.02 ; 1.83)$. The online stress management interventions differed in training time and training frequency. Training time ranged from 2 to 20 hours, training frequency ranged from 3 to 12 sessions. See Table 2 for all measures and outcomes of the 18 intervention studies, see Table 3 for details on stress measurement instruments, the means of pre and post measurements for these instruments and effect sizes. See table 4 for training time and frequency of the 18 intervention studies.

\subsection{Sustainability of Online Stress Management Training Interventions Effects}

In total, 10 of the 18 online stress management interventions used follow-up measurements to assess the sustainability of intervention effects. Heber et al. (2016) evaluated the intervention effects at three measurement points; 7 weeks, 6 months and 12 months after the intervention. The intervention turned out to be very effective in reducing stress levels and in improving different wellbeing outcomes such as work engagement and mental health. Other studies evaluated the intervention effects at two measurement points. Within the studies of Jung et al. (2016), Cieslack et al. (2016) and Ruwaard et al. (2007) all outcomes with significant effects remained significant at the follow-up measurement, respectively 8 weeks, 2 months and 3 years after the intervention. The studies of Ebert et al. (2016a; 2016b) were very effective, since almost all results remained significant at the follow-up measurement, 6 months after the intervention. The study of Eisen et al. (2008) revealed non-significant effects at the follow-up measurement, 1 month after the intervention.

Finally, the study of Yamagishi et al. (2008) was effective in relation to significantly improving career identity knowledge and impact on organizations 9 weeks after the intervention. However, the study by Yamagishi et.al, (2008) did not reveal positive effects on enhancing well-being or reducing job stress. In sum, of the 10 online stress management intervention studies that used follow-up measurements to assess the sustainability of intervention effects, 6 turn out to be effective to enhance employee well-being over a longer period of time. The time investment for the 6 online stress management interventions with sustainable intervention effects ranged between 3 and 17.5 hours. The most sustainable effects were found for the web- and mobile-based stress management training by Heber et al. (2016), the time investment for their online stress management training was 8.5 hour. See Table 5 for an overview of the study variables, the measurement points and frequencies of the 10 intervention studies that used follow-up measurements to assess the sustainability of intervention effects. 


\section{Macrothink}

International Journal of Learning and Development

ISSN 2164-4063

Table 2. Characteristics of the Online Stress Management Interventions

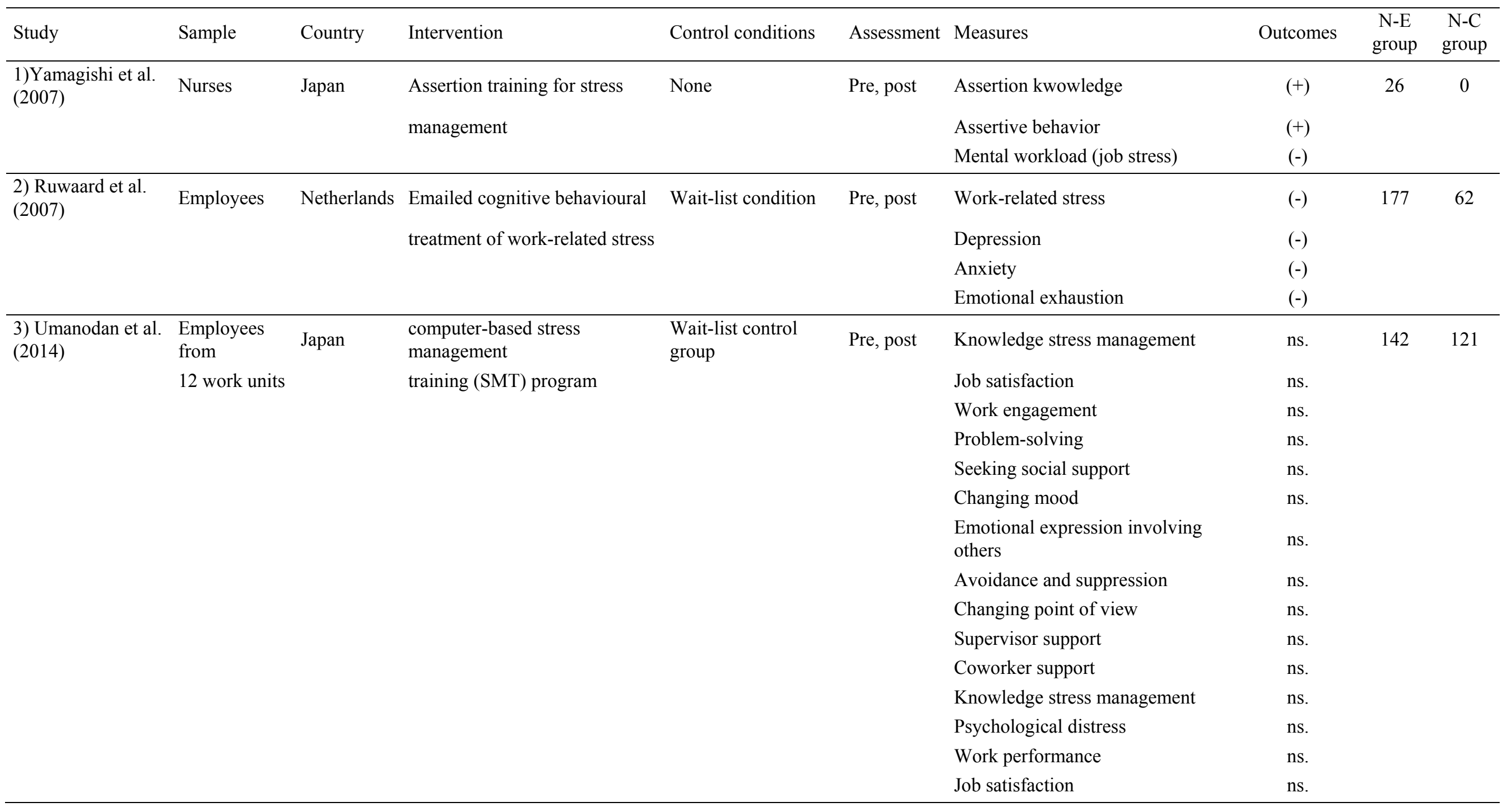




\begin{tabular}{|c|c|c|c|c|c|c|c|c|c|}
\hline $\begin{array}{l}\text { 4) Zetterqvistc et } \\
\text { al. (2003) }\end{array}$ & Employees & Sweden & $\begin{array}{l}\text { Internet-based self-help stress } \\
\text { management program }\end{array}$ & $\begin{array}{l}\text { Wait-list control } \\
\text { group }\end{array}$ & Pre, post & $\begin{array}{l}\text { Perceived stress } \\
\text { Hospital anxiety \& depression }\end{array}$ & $\begin{array}{l}(-) \\
(-)\end{array}$ & 63 & 23 \\
\hline $\begin{array}{l}\text { 5) Ebert et al. } \\
\text { (2016) }\end{array}$ & Employees & Germany & $\begin{array}{l}\text { Self-guided internet-based and } \\
\text { mobile-based stress } \\
\text { management intervention }\end{array}$ & $\begin{array}{l}\text { Wait-list control } \\
\text { group }\end{array}$ & Pre, post & $\begin{array}{l}\text { Perceived stress } \\
\text { Depression } \\
\text { Anxiety } \\
\text { Presenteeism } \\
\text { Emotional exhaustion } \\
\text { Sleeping problems } \\
\text { Worrying } \\
\text { Quality of life } \\
\text { Psychological detachment } \\
\text { Emotion regulation skill } \\
\text { Absenteeism } \\
\text { Work engagement }\end{array}$ & $\begin{array}{l}(-) \\
(-) \\
(-) \\
(+) \\
(-) \\
(-) \\
(-) \\
(+) \\
(+) \\
(+) \\
\text { ns. } \\
\text { ns. }\end{array}$ & 131 & 132 \\
\hline $\begin{array}{l}\text { 6) Ebert et al. } \\
(2016)\end{array}$ & $\begin{array}{l}\text { Employees } \\
\text { large health } \\
\text { insurance } \\
\text { company }\end{array}$ & Germany & $\begin{array}{l}\text { Internet- and mobile-based } \\
\text { stress management (cognitive } \\
\text { behavioural therapy) }\end{array}$ & $\begin{array}{l}\text { Wait-list control } \\
\text { group }\end{array}$ & Pre, post & $\begin{array}{l}\text { Perceived stress } \\
\text { Depression } \\
\text { Anxiety } \\
\text { Emotional exhaustion } \\
\text { Sleeping problems } \\
\text { Worrying } \\
\text { Quality of life } \\
\text { Psychological detachment } \\
\text { Emotion regulation skill } \\
\text { Presenteeism } \\
\text { Absenteeism } \\
\text { Work engagement }\end{array}$ & $\begin{array}{l}(-) \\
(-) \\
(-) \\
(-) \\
(-) \\
(-) \\
(+) \\
(+) \\
(+) \\
\text { ns. } \\
\text { ns. } \\
\text { ns. }\end{array}$ & 132 & 131 \\
\hline
\end{tabular}




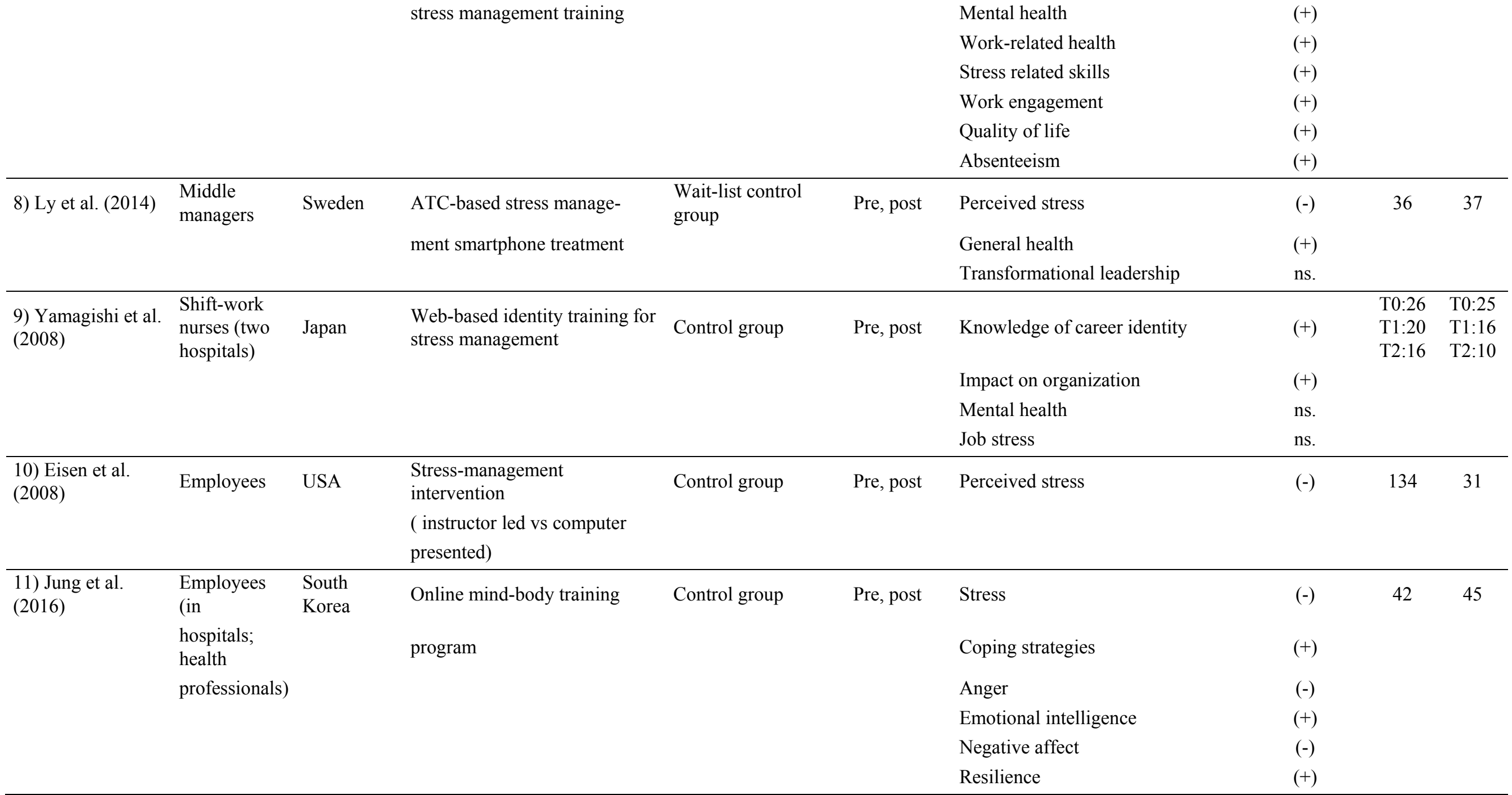




\section{Macrothink \\ Institute ${ }^{\text {mM }}$}

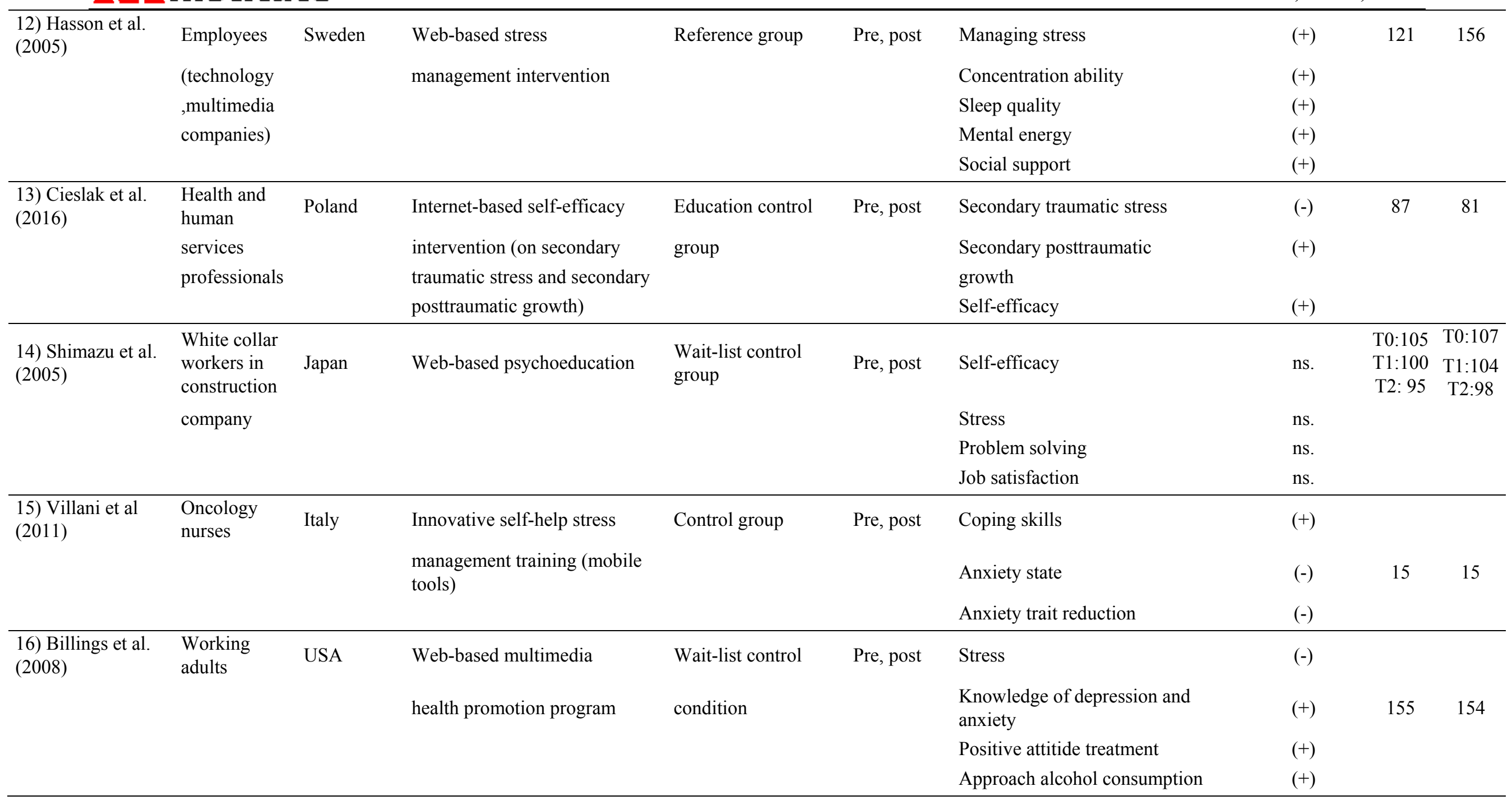




\section{Macrothink \\ Institute ${ }^{m}$}

International Journal of Learning and Development

ISSN 2164-4063

2018, Vol. 8, No. 3

\begin{tabular}{|c|c|c|c|c|c|c|c|c|c|}
\hline $\begin{array}{l}\text { 17) van Straten et } \\
\text { al. (2008) }\end{array}$ & Employees & Netherlands & $\begin{array}{l}\text { Web-based self-help } \\
\text { intervention } \\
\text { (mental health) }\end{array}$ & $\begin{array}{l}\text { Wait-list control } \\
\text { group }\end{array}$ & Pre, post & $\begin{array}{l}\text { Depression } \\
\text { Anxiety } \\
\text { Quality of life } \\
\text { Work related stress }\end{array}$ & $\begin{array}{l}(-) \\
(-) \\
\text { ns. } \\
\text { ns. }\end{array}$ & 107 & 106 \\
\hline $\begin{array}{l}\text { 18) Wolever et al. } \\
\text { (2012) }\end{array}$ & $\begin{array}{l}\text { Employees } \\
\text { (US) }\end{array}$ & USA & $\begin{array}{l}\text { Virtual online classroom } \\
\text { intervention }\end{array}$ & Control group & Pre, post & $\begin{array}{l}\text { Depression } \\
\text { Stress } \\
\text { Mindfulness }\end{array}$ & $\begin{array}{l}\text { ns. } \\
(-) \\
(+)\end{array}$ & 52 & 44 \\
\hline
\end{tabular}

All reported results significant to $\mathrm{p}<.05$ (or lower). (+) significant increase; (-) significant decrease; ns. No significant effects; $\mathrm{N}-\mathrm{E}$ group $=\mathrm{N}$ of experimental group; $\mathrm{N}-\mathrm{C}$ group $=\mathrm{N}$ of experimental group

Table 3. Stress measurement instruments, means of pre and post measurements and effect sizes.

\begin{tabular}{|c|c|c|c|c|c|c|}
\hline Study & Instruments & Pre (T0) & Post (T1) & Post (T2) & Outcomes & Effect sizes \\
\hline 1)Yamagishi et al. (2007) & Unknown sub-scale of job stress & $\mathrm{m}=2.21$ & $\mathrm{~m}=2.11$ & N/A & ns. & ns. \\
\hline 2) Ruwaard et al. (2007) & $\begin{array}{l}\text { Stress-subscale of Depression Anxiety } \\
\text { Stress Scales (DASS-42) }\end{array}$ & $\begin{array}{l}E: m=19.4 \\
C: m=19.7\end{array}$ & $\begin{array}{l}E: m=12.0 \\
C: m=17.6\end{array}$ & N/A & $(-)$ & $\begin{array}{l}\text { Within: } E: \mathrm{d}=0.6 \\
\text { Within: } \mathrm{C}: \mathrm{d}=0.2 \\
\text { Between: } \mathrm{d}=0.6\end{array}$ \\
\hline \multirow[t]{2}{*}{$\begin{array}{l}\text { 3) Umanodan et al. } \\
\text { (2014) }\end{array}$} & Unknown 'coping with stress' questions & $\begin{array}{l}\text { E: } m=2.8 \\
C: m=3.0\end{array}$ & $\begin{array}{l}\text { E: } m=3.2 \\
\text { C: } m=3.0\end{array}$ & $\begin{array}{l}\text { E: } \mathrm{m}=3.0 \\
\text { C: } \mathrm{m}=2.8\end{array}$ & $(+) /(-)$ & $\begin{array}{l}\text { T1: } \mathrm{d}=0.19 \\
\text { T2: } d=0.19\end{array}$ \\
\hline & Brief Job Stress Questionnaire & $\begin{array}{l}E: m=2.0 \\
C: m=2.1\end{array}$ & $\begin{array}{l}E: m=1.9 \\
C: m=2.0\end{array}$ & $\begin{array}{l}E: m=2.0 \\
C: m=2.0\end{array}$ & ns. & ns. \\
\hline $\begin{array}{l}\text { 4) Zetterqvist et al. } \\
\text { (2003) }\end{array}$ & Perceived Stress Scale & $\begin{array}{l}E: m=32.91 \\
C: m=33.17\end{array}$ & $\begin{array}{l}E: m=24.48 \\
C: m=28.88\end{array}$ & N/A & $(-)$ & N/A \\
\hline 5) Ebert et al. (2016) & Perceived Stress Scale-10 & $\begin{array}{l}E: m=25.7 \\
C: m=26.1\end{array}$ & $\begin{array}{l}\mathrm{E}: \mathrm{m}=18.1 \\
\mathrm{C}: \mathrm{m}=23.4\end{array}$ & $\begin{array}{l}\mathrm{E}: \mathrm{m}=17.5 \\
\mathrm{C}: \mathrm{m}=21.8\end{array}$ & $(-)$ & $\begin{array}{l}\text { T1: } d=0.96 \\
T 2: d=0.65\end{array}$ \\
\hline
\end{tabular}




\section{Macrothink \\ istinte}

\section{6) Ebert et al. (2016)}

7) Heber et al. (2016)

Perceived Stress Scale-10

$\mathrm{E}: \mathrm{m}=25.89$

$\mathrm{C}: \mathrm{m}=25.15$

$E: m=25.21$

$\mathrm{C}: \mathrm{m}=25.31$
International Journal of Learning and Development ISSN 2164-4063 2018, Vol. 8, No. 3

$E: m=18.79$

$\mathrm{E}: \mathrm{m}=17.05$

$\mathrm{C}: \mathrm{m}=23.33$

$\mathrm{C}: \mathrm{m}=22.24$

(-) $\quad$ T1: $d=0.79$

\begin{tabular}{|c|c|c|c|c|c|c|}
\hline 7) Heber et al. (2016) & Perceived Stress Scale-10 & $\mathrm{C}: \mathrm{m}=25.15$ & $C: m=22.96$ & $\mathrm{C}: \mathrm{m}=22.10$ & $(-)$ & $\begin{array}{l}\text { Within: } 10-\mathrm{T} 1: \mathrm{C}: \mathrm{d}= \\
0.41 \\
\text { Within: T0-T2: C: } \mathrm{d}= \\
0.60\end{array}$ \\
\hline \multirow[t]{2}{*}{ 8) Ly et al. (2014) } & $\begin{array}{l}\text { Perceived Stress Scale-14 } \\
\text { Perceived Stress Scale-10 }\end{array}$ & $\begin{array}{l}E: m=24.33 \\
C: m=24.49\end{array}$ & $\begin{array}{l}E: m=19.52 \\
C: m=23.29\end{array}$ & N/A & $(-)$ & $\begin{array}{l}\text { Between: } d=0.50 \\
\text { Within: } E: d=0.62 \\
\text { Within: } C: d=0.17\end{array}$ \\
\hline & Brief Job Stress Questionnaire & $\begin{array}{l}E: m=(2.41 ; 2.97) \\
C: m=(2.33 ; 2.90)\end{array}$ & $\begin{array}{l}E: m=(2.18 ; 2.83) \\
C: m=(2.40 ; 2.79)\end{array}$ & $\begin{array}{l}E: m=(2.17 ; 2.94) \\
C: m=(2.23 ; 2.93)\end{array}$ & ns. & ns. \\
\hline 10) Eisen et al. (2008) & Stress Pulse Record & $\begin{array}{l}\text { Session } 1: \\
E: m=42.9 \\
C: m=45.8 \\
\text { Session } 2: \\
E: m=46.7 \\
C: m=53.3\end{array}$ & $\begin{array}{l}\text { Session } 1: \\
E: m=21.7 \\
C: m=38.3 \\
\text { Session } 2: \\
E: m=21.1 \\
C: m=33.3\end{array}$ & $\mathrm{~N} / \mathrm{A}$ & $(-)$ & $\mathrm{N} / \mathrm{A}$ \\
\hline 11) Jung et al. (2016) & Stress Response Inventory & $\begin{array}{l}E: m=28.79 \\
C: m=26.80\end{array}$ & $\begin{array}{l}E: m=25.79 \\
C: m=34.09\end{array}$ & $\begin{array}{l}E: m=24.62 \\
C: m=37.31\end{array}$ & $(+)$ & $\mathrm{N} / \mathrm{A}$ \\
\hline 12) Hasson et al. (2005) & Managing stress & $\begin{array}{l}E: m=60 \\
C: m=60\end{array}$ & $\begin{array}{l}E: m=63 \\
C: m=58\end{array}$ & N/A & $(+)$ & $\mathrm{N} / \mathrm{A}$ \\
\hline
\end{tabular}




\section{Macrothink}

International Journal of Learning and Development ISSN 2164-4063 2018, Vol. 8, No. 3

Between: $\mathrm{T} 1: \mathrm{d}=0.49$ Between: T2: $\mathrm{d}=0.08$

Within: T0-T1: $E$ : $d=0.29$

13) Cieslak et al. (2016) Secondary Traumatic Stress Scale

Within: T1-T2: E: $\mathrm{d}=$

$-0.02$

Within: T0-T1: C: $\mathrm{d}=$ 0.08

Within: T1-T2: $\mathrm{C}: \mathrm{d}=$ 0.15

\begin{tabular}{|c|c|c|c|c|c|c|}
\hline 14) Shimazu et al. (2005) & Brief Job Stress Questionnaire & $\mathrm{N} / \mathrm{A}$ & N/A & $\begin{array}{l}E: \Delta m=-1.07 \\
C: \Delta m=-0.72\end{array}$ & ns. & ns. \\
\hline & Brief Job Stress Questionnaire & $\mathrm{N} / \mathrm{A}$ & N/A & $\begin{array}{l}E: \Delta m=-0.77 \\
C: \Delta m=-0.27\end{array}$ & ns. & ns. \\
\hline 15) Villani et al (2011) & State Trait Anxiety Inventory & $\begin{array}{l}E: m=9.429 \\
C: m=3.077\end{array}$ & $\begin{array}{l}E: m=5.067 \\
C: m=0.214\end{array}$ & N/A & $(-)$ & N/A \\
\hline 16) Billings et al. (2008) & Symptoms of Distress Scale & $\begin{array}{l}E: m=17.52 \\
C: m=16.81\end{array}$ & $\begin{array}{l}E: m=16.03 \\
C: m=16.50\end{array}$ & N/A & $(-)$ & $\mathrm{N} / \mathrm{A}$ \\
\hline \multirow[t]{3}{*}{$\begin{array}{l}\text { 17) van Straten et al. } \\
\text { (2008) }\end{array}$} & Maslach Burnout Inventory & $\begin{array}{l}E: m=2.9 \\
C: m=2.8\end{array}$ & $\begin{array}{l}E: m=2.5 \\
C: m=2.8\end{array}$ & N/A & ns. & N/A \\
\hline & Maslach Burnout Inventory & $\begin{array}{l}E: m=3.2 \\
C: m=3.4\end{array}$ & $\begin{array}{l}E: m=3.5 \\
C: m=3.2\end{array}$ & N/A & ns. & N/A \\
\hline & Maslach Burnout Inventory & $\begin{array}{l}E: m=2.4 \\
C: m=2.4\end{array}$ & $\begin{array}{l}E: m=2.3 \\
C: m=2.6\end{array}$ & N/A & ns. & $\mathrm{N} / \mathrm{A}$ \\
\hline 18) Wolever et al. (2012) & Perceived Stress Scale-10 & $\begin{array}{l}E: m=24.52 \\
C: m=24.85\end{array}$ & $\begin{array}{l}E: m=14.91 \\
C: m=16.94\end{array}$ & N/A & $(-)$ & $\eta^{2}=0.02$ \\
\hline
\end{tabular}

All reported results significant to $\mathrm{p}<.05$ (or lower). $(+)$ significant increase; (-) significant decrease; ns. non-significant $; \mathrm{N}-\mathrm{E}$ group $=\mathrm{N}$ of experimental group; $\mathrm{N} / \mathrm{A}=$ not available;

$\mathrm{E}=$ experimental group $\mathrm{C}=$ control group 


\section{Macrothink}

International Journal of Learning and Development

ISSN 2164-4063

Table 4. Online Stress Management Intervention training time and frequency

\begin{tabular}{|c|c|c|c|c|}
\hline Study & Training frequency & Training hours & Related outcomes & \\
\hline \multirow[t]{5}{*}{ 1)Yamagishi et al. (2007) } & $70 \mathrm{mn} /$ week/3weeks & 3,3 & Assertion kwowledge & $(+)$ \\
\hline & & & Assertive behavior & $(+)$ \\
\hline & & & Assertive mind & ns. \\
\hline & & & Job stress & ns. \\
\hline & & & Depression & ns. \\
\hline \multirow[t]{4}{*}{ 2) Ruwaard et al. (2007) } & $30 \mathrm{mn} /$ day/7weeks & 17,5 & Work-related stress & $(-)$ \\
\hline & & & Depression & $(-)$ \\
\hline & & & Anxiety & $(-)$ \\
\hline & & & Emotional exhaustion & $(-)$ \\
\hline \multirow[t]{10}{*}{ 3) Umanodan et al. (2014) } & $30 \mathrm{mn} /$ week/7weeks & 3,5 & Knowledge stress management & ns. \\
\hline & & & Supervisor support & ns. \\
\hline & & & Coworker support & ns. \\
\hline & & & Knowledge stress management & ns. \\
\hline & & & Psychological distress & ns. \\
\hline & & & Work performance & ns. \\
\hline & & & Job satisfaction & ns. \\
\hline & & & Work engagement & ns. \\
\hline & & & Problem-solving & ns. \\
\hline & & & Seeking social support & ns. \\
\hline
\end{tabular}




\begin{tabular}{|c|c|c|c|c|}
\hline & & & Changing mood & ns. \\
\hline & & & Emotional expression involving others & ns. \\
\hline & & & Avoidance and suppression & ns. \\
\hline & & & Changing point of view & ns. \\
\hline \multirow[t]{4}{*}{ 4) Zetterqvistc et al. (2003) } & $40 \mathrm{mn} / 6 \mathrm{modelules}$ & 4 & Perceived stress & $(-)$ \\
\hline & & & Hospital anxiety and depression & $(-)$ \\
\hline & & & Perceived family support & ns. \\
\hline & & & Perceived friends support & ns. \\
\hline \multirow[t]{12}{*}{ 5) Ebert et al. (2016) } & $60 \mathrm{mn} / \mathrm{session} / 4 \mathrm{weeks}$ & 8 & Perceived stress & $(-)$ \\
\hline & & & Depression & $(-)$ \\
\hline & & & Anxiety & $(-)$ \\
\hline & & & Presenteeism & $(+)$ \\
\hline & & & Emotional exhaustion & $(-)$ \\
\hline & & & Sleeping problems & $(-)$ \\
\hline & & & Worrying & $(-)$ \\
\hline & & & Quality of life & $(-)$ \\
\hline & & & Psychological detachment & $(+)$ \\
\hline & & & Emotion regulation skill & $(+)$ \\
\hline & & & Absenteeism & ns. \\
\hline & & & Work engagement & ns. \\
\hline \multirow[t]{2}{*}{ 6) Ebert et al. (2016) } & $60 \mathrm{mn} / 8$ sessions & 8 & Perceived stress & $(-)$ \\
\hline & & & Depression & $(-)$ \\
\hline
\end{tabular}




\section{Interatinak \\ Institute ${ }^{\text {TM }}$}

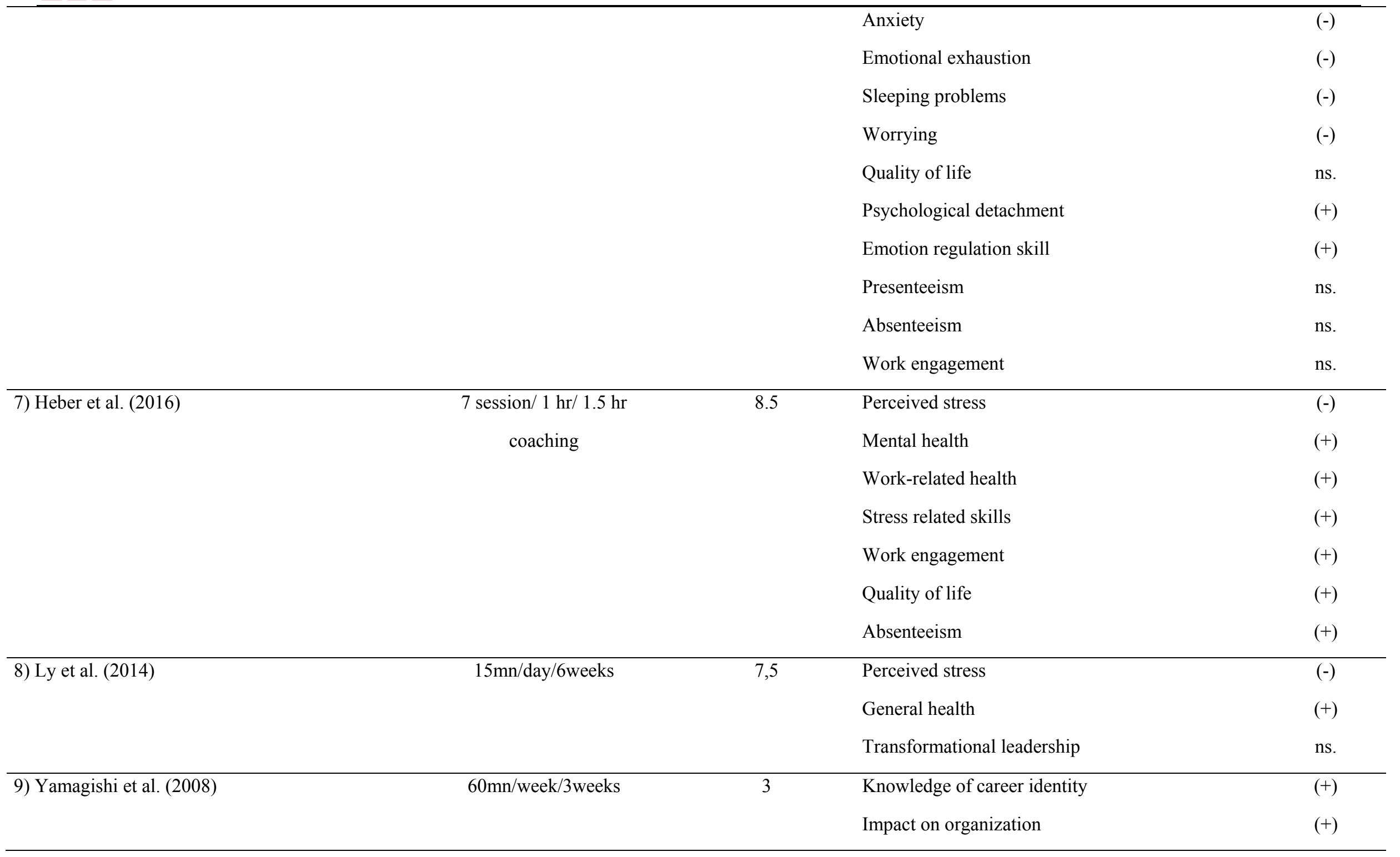




\section{Macrothink Minstitute}

\begin{tabular}{|c|c|c|c|c|}
\hline & & & Mental health & ns. \\
\hline & & & Job stress & ns. \\
\hline \multirow[t]{3}{*}{ 10) Eisen et al. (2008) } & $55 \mathrm{mn} /$ week/2weeks \& & 4 & Perceived stress & $(-)$ \\
\hline & $10 \mathrm{mn} / \mathrm{session} / 2$ sessions & & Subjective distress & ns. \\
\hline & & & Control over stress & ns. \\
\hline \multirow[t]{6}{*}{ 11) Jung et al. (2016) } & $10 \mathrm{mn} /$ day/8weeks & 7 & Stress & $(-)$ \\
\hline & & & Coping strategies & $(+)$ \\
\hline & & & Anger & $(-)$ \\
\hline & & & Emotional intelligence & $(+)$ \\
\hline & & & Negative affect & $(-)$ \\
\hline & & & Resilience & $(+)$ \\
\hline \multirow[t]{5}{*}{ 12) Hasson et al. (2005) } & & 20 & Managing stress & $(+)$ \\
\hline & & & Concentration ability & $(+)$ \\
\hline & & & Sleep quality & $(+)$ \\
\hline & & & Mental energy & $(+)$ \\
\hline & & & Social support & $(+)$ \\
\hline \multirow[t]{3}{*}{ 13) Cieslak et al. (2016) } & 4 sessions/ 4 weeks & & Secondary traumatic stress & $(-)$ \\
\hline & & & Secondary posttraumatic growth & $(+)$ \\
\hline & & & Self-efficacy & $(+)$ \\
\hline \multirow[t]{3}{*}{ 14) Shimazu et al. (2005) } & 5 modules & & Self-efficacy & ns. \\
\hline & & & Stress & ns. \\
\hline & & & Problem solving & ns. \\
\hline
\end{tabular}

International Journal of Learning and Development ISSN $2164-4063$ 2018, Vol. 8, No. 3 


\section{Interatinat Mnstitute"}

\begin{tabular}{|c|c|c|c|c|}
\hline & & & Job satisfaction & ns. \\
\hline \multirow[t]{3}{*}{ 15) Villani et al. (2011) } & $15 \mathrm{mn} / \mathrm{session} / 8$ session & 2 & Coping skills & $(+)$ \\
\hline & & & Anxiety state & $(-)$ \\
\hline & & & Anxiety trait reduction & $(-)$ \\
\hline \multirow[t]{4}{*}{ 16) Billings et al. (2008) } & & & Stress & $(-)$ \\
\hline & & & Knowledge of depression and anxiety & $(+)$ \\
\hline & & & Positive attitide treatment & $(+)$ \\
\hline & & & Approach alcohol consumption & $(+)$ \\
\hline \multirow[t]{4}{*}{ 17) van Straten et al. (2008) } & $45 \mathrm{mn} /$ week/4weeks & 3 & Depression & $(-)$ \\
\hline & & & Anxiety & $(-)$ \\
\hline & & & Quality of life & ns. \\
\hline & & & Work related stress & ns. \\
\hline \multirow[t]{3}{*}{ 18) Wolever et al. (2012) } & $14 \mathrm{hr} / 12$ weeks & 14 & Depression & (ns.) \\
\hline & & & Stress & $(-)$ \\
\hline & & & Mindfulness & $(+)$ \\
\hline
\end{tabular}

International Journal of Learning and Development ISSN 2164-4063 2018, Vol. 8, No. 3

All reported results significant to $\mathrm{p}<.05$ (or lower). (+) significant increase; (-) significant decrease; ns. non-significant 


\section{Macrothink \\ institute ${ }^{\mathrm{TM}}$}

International Journal of Learning and Development

ISSN 2164-4063

Table 5. Sustainability of the Online Stress Management Intervention Effects

\begin{tabular}{|c|c|c|c|c|}
\hline Study & Training frequency & Training hours & Related outcomes & \\
\hline \multirow[t]{5}{*}{ 1)Yamagishi et al. (2007) } & 70mn/week/3weeks & 3,3 & Assertion kwowledge & $(+)$ \\
\hline & & & Assertive behavior & $(+)$ \\
\hline & & & Assertive mind & ns. \\
\hline & & & Job stress & ns. \\
\hline & & & Depression & ns. \\
\hline \multirow[t]{4}{*}{ 2) Ruwaard et al. (2007) } & $30 \mathrm{mn} /$ day $/ 7$ weeks & 17,5 & Work-related stress & $(-)$ \\
\hline & & & Depression & $(-)$ \\
\hline & & & Anxiety & $(-)$ \\
\hline & & & Emotional exhaustion & $(-)$ \\
\hline \multirow[t]{14}{*}{ 3) Umanodan et al. (2014) } & $30 \mathrm{mn} /$ week/7weeks & 3,5 & Knowledge stress management & ns. \\
\hline & & & Supervisor support & ns. \\
\hline & & & Coworker support & ns. \\
\hline & & & Knowledge stress management & ns. \\
\hline & & & Psychological distress & ns. \\
\hline & & & Work performance & ns. \\
\hline & & & Job satisfaction & ns. \\
\hline & & & Work engagement & ns. \\
\hline & & & Problem-solving & ns. \\
\hline & & & Seeking social support & ns. \\
\hline & & & Changing mood & ns. \\
\hline & & & Emotional expression involving others & ns. \\
\hline & & & Avoidance and suppression & ns. \\
\hline & & & Changing point of view & ns. \\
\hline \multirow[t]{2}{*}{ 4) Zetterqvistc et al. (2003) } & $40 \mathrm{mn} / 6$ modelules & 4 & Perceived stress & $(-)$ \\
\hline & & & Hospital anxiety and depression & $(-)$ \\
\hline
\end{tabular}




\section{Macrothink A Instiute}

5) Ebert et al. (2016)

6) Ebert et al. (2016)

7) Heber et al. (2016)
$60 \mathrm{mn} / \mathrm{session} / 4$ weeks

$60 \mathrm{mn} / 8$ sessions coaching
International Journal of Learning and Development

ISSN 2164-4063 2018, Vol. 8, No. 3

Perceived family support

ns.

Perceived friends support

Perceived stress

$(-)$

Depression

Anxiety

Presenteeism

Emotional exhaustion

Sleeping problems

Worrying

Quality of life

Psychological detachment

Emotion regulation skill

Absenteeism

Work engagement

Perceived stress

Depression

Anxiety

Emotional exhaustion

Sleeping problems

Worrying

Quality of life

Psychological detachment

Emotion regulation skill

Presenteeism

$(-)$

$(-)$

(+)

(-)

$(-)$

(-)

$(-)$

(+)

(+)

ns.

ns.

(-)

$(-)$

$(-)$

(-)

$(-)$

ns.

(+)

$(+)$

ns.

Absenteeism

ns.

Work engagement

ns.

Perceived stress

$(-)$

Mental health 


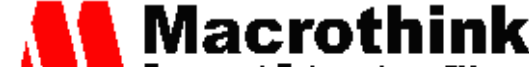 nastatue}

8) Ly et al. (2014)

9) Yamagishi et al. (2008)

10) Eisen et al. (2008)

11) Jung et al. (2016)

12) Hasson et al. (2005)

13) Cieslak et al. (2016)
$15 \mathrm{mn} /$ day/6weeks

$60 \mathrm{mn} /$ week/3weeks

$55 \mathrm{mn} /$ week/2weeks \&

$10 \mathrm{mn} /$ session $/ 2$ sessions

$10 \mathrm{mn} /$ day $/ 8$ weeks

sessions/ 4 weeks
International Journal of Learning and Development

ISSN 2164-4063 2018, Vol. 8, No. 3

Work-related health

Stress related skills

Work engagement

Quality of life

$(+)$

Perceived stress

General health

Transformational leadership

Knowledge of career identity

Impact on organization

$(+)$

Mental health

ns.

Job stress

Perceived stress

Subjective distress

Control over stress

Stress

Coping strategies

Anger

Emotional intelligence

Negative affect

Resilience

$20 \quad$ Managing stress

Concentration ability

Sleep quality

Mental energy

Social support

$(+)$

Secondary traumatic stress

$(-)$

(+) 


\section{Interatibat 1. Instinte}

14) Shimazu et al. (2005)

15) Villani et al. (2011)

16) Billings et al. (2008)

17) van Straten et al. (2008)

18) Wolever et al. (2012)
$15 \mathrm{mn} / \mathrm{session} / 8$ session

$45 \mathrm{mn} /$ week/4weeks

$14 \mathrm{hr} / 12$ weeks
International Journal of Learning and Development

ISSN 2164-4063 2018, Vol. 8, No. 3

Self-efficacy

$(+)$

Self-efficacy

ns.

Stress

ns.

Problem solving

Job satisfaction

2

Coping skills

ns.

ns.

Anxiety state

$(+)$

$(-)$

Anxiety trait reduction

Stress

Knowledge of depression and anxiety

Positive attitide treatment

Approach alcohol consumption

Depression

Anxiety

Quality of life

Work related stress

ns.

Depression

(ns.)

Stress

$(-)$

$(+)$

All reported results significant to $\mathrm{p}<.05$ (or lower). $(+)$ significant increase; (-) significant decrease; ns. non-significant 


\section{Discussion}

This study aimed to contribute to the literature on online training interventions by conducting a literature review study on the impact of online stress management interventions aimed at fostering employee well-being. We examined both the effectiveness of 18 online stress management interventions and the sustainability of the intervention effects. Based on the results of this review, online stress management interventions turn out to be a promising tool to enhance employee well-being. In addition, more than half of the studies who measured intervention effects two or three times, revealed that intervention effects were sustainable over time. To our knowledge this is the first literature review study examining the effectiveness and sustainability of this type of organizational interventions on employee well-being. In the next section, we discuss the most important contributions of our study.

\subsection{Theoretical Contributions}

A first theoretical contribution of this article is that it adds to the existing intervention literature by being the first to offer a concise overview of the effectiveness of online stress management interventions in enhancing employees' well-being. Although earlier empirical reviews showed the potential of online interventions, (Mitchell et al., 2010; Wolever et al., 2012) until now no study had examined the effectiveness of online organizational stress management interventions in enhancing employees' well-being. Our review offers an overview of the different types of intervention studies conducted over the last fifteen years, including the intervention characteristics, intervention outcomes and the sustainability of the outcomes. Therefore, this study may help researchers to gain a better understanding of intervention design, quality and effectiveness. Second, the large total number of 3085 participants who work in different sectors in all parts of the world makes the literature review sample representative and relevant. In addition, by limiting the scope of the review to the years 2002-2017, it also gives a state-of-the-art overview that can be used as a base for future online intervention research.

\subsection{Limitations and Research Issues}

Besides the theoretical contributions, some limitations of our study need to be acknowledged. There are some factors that should still be kept in mind when interpreting the conclusions presented in this article. First, we limited our scope to online stress management interventions, rather than having a broader search strategy. Although this scope helped to gain a deep understanding of outcomes, effectiveness and sustainability, we cannot compare them with other types of online interventions aimed at enhancing employee well-being. Future studies might consider including other types of online organizational interventions like online mindfulness interventions, online mental well-being interventions and/or online work engagement interventions as well. Second, in most of the studies examined in this review, the outcomes were based on participants' self-reports, rather than more 'objective' measures. Although this is not something that was caused by our methodology specifically, it does mean that the results presented in this article should be interpreted with potential self-report bias in mind. However, employees' evaluations of their well-being may be subjective, and self-reports may be the best method to capture these perceptions and feelings (Sousa-Poza, \& Sousa-Poza, 2000). In addition to measuring well-being and stress via questionnaires, future studies may 
consider including measures of participants' levels of cortisol. Third, because of the rigor of our methodology, only a relatively small number of articles ended up being discussed in this study. However, since the final selection has been extensively screened beforehand in terms of methodological quality and relevance, we feel that the lack of quantity is mitigated by having a high-quality sample instead.

Beyond the limitations, there are also some research issues that need to be acknowledged. First, it is remarkable that in at least half of the studies, the control group showed a significant decrease in stress as well, albeit not as strong as the experimental group. In most studies, there were no significant differences between the control group and the experimental group(s) in terms of baseline statistics, with the exception of the study by Villani et al. (2013), where the experimental group scored higher on anxiety than the control group at T1. Further, the inconsistencies in the measurement of stress among the 18 studies challenges the comparability and interpretation of the outcomes. Therefore, we recommend that instead of only making general conclusions about all the studies together, to also take a closer look at the individual differences between studies and value the unique characteristics of each study separately. In addition, only 9 of the 18 studies reported significant estimates of effect sizes. With the exception of 1 study, they all reported Cohen's $d$. Only 4 studies distinguished between 'within group' and 'between group' effect sizes, where the effect sizes for the experimental groups varied widely $(-0.02 ; 1.83)$. All in all, although there is evidence that online stress interventions reduce (perceived) stress, we have to take some caution into account in interpreting and generalizing the results.

\subsection{Practical Implications}

Besides theoretical contributions, the findings of this study also have practical implications for organizations, managers and employees. We suggest that organizations that aim to enhance employee well-being may find an efficient and effective solution in online stress management interventions. As online interventions turn out to be (cost) effective and can easily be tailored to individual needs (Stralen et al., 2011; Napolitano et al., 2003), these interventions show potential for all types of organizations. For organizations with small learning and development and/or mental health policy budgets, these type of interventions offer an affordable opportunity to foster employee well-being. The findings of this study may also have implications for HR(D) departments in relation to their learning and development and/or health policies and the programs they facilitate in the organization. Managers can stimulate employees to participate in online stress management interventions by sharing the positive contributions they may have on their well-being. Based on the review, the online cognitive-behavioral stress management interventions turn out to be the most effective in decreasing employees' stress and in improving employees' mental health, work-related health, work engagement and quality of life. In addition, these effects turn out to be sustainable up to 1 year after completing the intervention. Thus, from a management perspective, it would be recommendable to (proactively) offer employees online cognitive-behavioral stress management interventions to enhance their well-being. However, it needs to be mentioned that employees own preferences, interest and learning motivations are aspects in learning and development that may play an important role in the actual effectiveness of interventions. As the results of the literature review revealed, all 
five sub types of online stress management interventions (Cognitive- behavioral based stress management interventions, Psycho-education based stress management interventions, Assertion training to manage stress levels, Career identity stress management training and Self-help based stress management interventions) have their own approach, focus, strengths and limitations. To help employees to select the type of intervention that has the most optimal fit with their needs and preferences, it is important to gain insights in their needs. Participants' input is not only essential to select the most effective intervention, this input also gives the opportunity to create a tailor made and personalized experience (for example Aikens et al. (2014)). For employees, this study underlines that online positive organizational interventions can support them to stay healthy and engaged in their job.

\subsection{Future Research and Conclusion}

Considering our limitations and implications, various suggestions for future research can be made. This literature review study not only gives a clear overview of the effectiveness of online stress management training interventions and sustainability of the intervention effects, but also reveals the need for future research. Because our search was limited to only including online stress management training interventions, it makes sense to broaden this scope in future research by including other types of online training interventions aimed at enhancing employee well-being as well. Most studies in this literature review present a description of the intervention design including the (estimated) time needed for participants to complete the intervention. However, we do need to mention that there is a lack of information on the time participants actually spend from start till finish. The newest technology and learning management systems can provide detailed information on participation time, for example on total time participants spend using the online intervention, which assignments of the interventions are completed, and how often participants logged into the different intervention chapters/elements. Insights in this information may help to gain a better understanding of differences in online intervention effectiveness.

Online stress management training interventions offer organizations an opportunity to actively enhance employees' well-being. As the outcomes of this study revealed, investing in this type of training interventions can be beneficial for both employees and organizations, on short and long term. Organizations who see online training interventions as a window of opportunities to invest in conditions for a healthy work environment, can proactively facilitate these interventions. By doing so, organizations take charge to create optimal conditions for a sustainable healthy workforce.

\section{Funding}

This research received no specific grant from any funding agency in the public, commercial, or not-for-profit sectors.

\section{References}

Aikens, K. A., Astin, J., Pelletier, K. R., Levanovich, K., Baase, C. M., Park, Y. Y., \& Bodnar, C. M. (2014). Mindfulness goes to work: Impact of an online workplace intervention. Journal of Occupational and Environmental Medicine, 56(7), 721-731. 
https://doi.org/10.1097/JOM.0000000000000209

Aldana, S. G. (2001). Financial impact of health promotion programs: a comprehensive review of the literature. The American Journal of Health Promotion, 15(5), 296-320. https://doi.org/10.4278/0890-1171-15.5.296

Avey, J. B., Wernsing, T. S., \& Luthans, F. (2008). Can positive employees help positive organizational change? Impact of psychological capital and emotions on relevant attitudes and behaviors. The journal of applied behavioral science, 44(1), 48-70. https://doi.org/10.1177/0021886307311470

Billings, D. W., Cook, R. F., Hendrickson, A., \& Dove, D. C. (2008). A web-based approach to managing stress and mood disorders in the workforce. Journal of occupational and environmental medicine/American College of Occupational and Environmental Medicine, 50(8), 960. https://doi.org/10.1097/JOM.0b013e31816c435b

Cieslak, R., Benight, C. C., Rogala, A., Smoktunowicz, E., Kowalska, M., Zukowska, K., \& Luszczynska, A. (2016). Effects of Internet-Based Self-Efficacy Intervention on Secondary Traumatic Stress and Secondary Posttraumatic Growth among Health and Human Services Professionals Exposed to Indirect Trauma. Frontiers in Psychology, 7. https://doi.org/10.3389/fpsyg.2016.01009

Cook, R., Billings, D., Hersch, R., Back, A., \& Hendrickson, A. (2007). A field test of a webbased workplace health promotion program to improve dietary practices, reduce stress, and increase physical activity: randomized controlled trial. Journal of medical Internet research, 9(2), e17. https://doi.org/10.2196/jmir.9.2.e17

Eastern Kentucky University (2016). Work related stress on employees health. Retrieved 27 November, 2017 from https://safetymanagement.eku.edu/resources/infographics/work-related-stress-on-employeeshealth/

Ebert, D. D., Heber, E., Berking, M., Riper, H., Cuijpers, P., Funk, B., \& Lehr, D. (2016a). Self-guided internet-based and mobile-based stress management for employees: results of a randomised controlled trial. Occupational and environmental medicine, 73(5), 315-323. https://doi.org/10.1136/oemed-2015-103269

Ebert, D. D., Lehr, D., Heber, E., Riper, H., Cuijpers, P., \& Berking, M. (2016b). Internet-and mobile-based stress management for employees with adherence-focused guidance: efficacy and mechanism of change. Scandinavian journal of work, environment \& health, 42(5), 382-394. https://doi.org/10.5271/sjweh.3573

Eisen, K. P., Allen, G. J., Bollash, M., \& Pescatello, L. S. (2008). Stress management in the workplace: A comparison of a computer-based and an in-person stress-management intervention. Computers in Human Behavior, 24(2), 486-496. https://doi.org/10.1016/j.chb.2007.02.003

EU-OSHA - European Agency for Safety and Health at Work, European survey of 
enterprises on new and emerging risks: managing safety and health at work, European Communities, Luxembourg, 2010. Retrieved 27 November, 2017, from https://osha.europa.eu/en/publications/reports/esener1_osh_management

Giga, S., Noblet, A., Faragher, B., \& Cooper, C. (2003). The UK Perspective: A Review of Research on Organisational Stress Management Interventions. Australian Psychologist, 38(2), 158-164. https://doi.org/10.1080/00050060310001707167

Hasson, D., Anderberg, U. M., Theorell, T., \& Arnetz, B. B. (2005). Psychophysiological effects of a web-based stress management system: a prospective, randomized controlled intervention study of IT and media workers [ISRCTN54254861]. BMC public health, 5(1), 78. https://doi.org/10.1186/1471-2458-5-78

Heber, E., Lehr, D., Ebert, D. D., Berking, M., \& Riper, H. (2016). Web-based and mobile stress management intervention for employees: a randomized controlled trial. Journal of medical Internet research, 18(1). https://doi.org/10.2196/jmir.5112

Jung, Y. H., Ha, T. M., Oh, C. Y., Lee, U. S., Jang, J. H., Kim, J., \& Kang, D. H. (2016). The Effects of an Online Mind-Body Training Program on Stress, Coping Strategies, Emotional Intelligence, Resilience and Psychological State. PloS one, 11(8), e0159841. https://doi.org/10.1371/journal.pone.0159841

Le Fevre, M., Kolt, G., Matheny, J. (2006). Eustress, distress and their interpretation in primary and secondary occupational stress management interventions: which way first? Journal of Managerial Psychology, 21(6), 547-565. https://doi.org/10.1108/02683940610684391

Ly, K. H., Asplund, K., \& Andersson, G. (2014). Stress management for middle managers via an acceptance and commitment-based smartphone application: A randomized controlled trial. Internet Interventions, 1(3), 95-101. https://doi.org/10.1016/j.invent.2014.06.003

Meyers, M.C., Van Woerkom, M., \& Bakker, A.B. (2013), The added value of the positive: A literature review of positive psychology interventions in organizations, European Journal of Work and Organizational Psychology, 22, 618-632. https://doi.org/10.1080/1359432X.2012.694689

Mitchell, J., Vella-Brodrick, D., \& Klein, B. (2010). Positive psychology and the internet: A mental health opportunity. Sensoria: A Journal of Mind, Brain \& Culture, 6(2), 30-41. https://doi.org/10.7790/ejap.v6i2.230

Napolitano, M., Fotheringham, M., Tate, D., Sciamanna, C., Leslie, E., Owen, N., Marcus, B. (2003). Evaluation of an Internet-based physical activity intervention: a preliminary investigation. Annals of Behavioral Medicine, 25, 92-99. https://doi.org/10.1207/S15324796ABM2502_04

Pace, R., Pluye, P., Bartlett, G., Macaulay, A. C., Salsberg, J., Jagosh, J., \& Seller, R. (2012). Testing the reliability and efficiency of the pilot Mixed Methods Appraisal Tool (MMAT) for systematic mixed studies review. International Journal of Nursing Studies, 49, 47-53. 
https://doi.org/10.1016/j.ijnurstu.2011.07.002

Pluye, P., \& Hong, Q. N. (2014). Combining the power of stories and the power of numbers: mixed methods research and mixed studies reviews. Annual Review of Public Health, 35, 29-45. https://doi.org/10.1146/annurev-publhealth-032013-182440

Pluye, P., Robert, E., Cargo, M., Bartlett, G., O'cathain, A., Griffiths, F., Rousseau, M. C. (2011). Proposal: A mixed methods appraisal tool for systematic mixed studies reviews (pp. 1-8). Retrieved on March 8, 2017 from $\mathrm{h}$ ttp://mixedmethodsappraisaltoolpublic.pbworks.com

Randolfi, E. A. (1997). Developing a stress management and relaxation center for the worksite. Worksite Health, 4(3), 40-44.

Riedell, J. E., Lynch, W., Baase, C., Hymel, P., \& Peterson, K. W. (2001). The effect of disease prevention and health promotion on workplace productivity: a literature review. The American Journal of Health Promotion, 15(3), 167-191. https://doi.org/10.4278/0890-1171-15.3.167

Ruwaard, J., Lange, A., Bouwman, M., Broeksteeg, J., \& Schrieken, B. (2007). E - Mailed standardized cognitive behavioural treatment of work-related stress: A randomized controlled trial. Cognitive Behaviour Therapy, 36(3), 179-192. https://doi.org/10.1080/16506070701381863

Schlager, M., Fusco, J., \& Schank, P. (2002). Evolution of an online education community of practice. In K. A. Renninger \& W. Shumar (Eds.), Building virtual communities: Learning and change in cyberspace (pp. 129-158). New York: Cambridge University Press. https://doi.org/10.1017/CBO9780511606373.010

Shimazu, A., Kawakami, N., Irimajiri, H., Sakamoto, M., \& Amano, S. (2005). Effects of web-based psychoeducation on self-efficacy, problem solving behavior, stress responses and job satisfaction among workers: a controlled clinical trial. Journal of occupational health, 47(5), 405-413. https://doi.org/10.1539/joh.47.405

Sousa-Poza, A., \& Sousa-Poza, A. A. (2000). Well-being at work: a cross-national analysis of the levels and determinants of job satisfaction. The journal of socio-economics, 29(6), 517-538. https://doi.org/10.1016/S1053-5357(00)00085-8

Stralen, M. M., de Vries, H., Mudde, A. N., Bolman, C., \& Lechner, L. (2011). The long-term efficacy of two computer-tailored physical activity interventions for older adults: Main effects and mediators. Health Psychology, 30, 442-452. https://doi.org/10.1037/a0023579

Thiart H, Lehr D, Ebert DD, Berking M, Riper H. (2015). Log in and breathe out: internet-based recovery training for sleepless employees with work-related strain - results of a randomized controlled trial. Scandinavian Journal of Work, Environment \& Health, 41(2), 164-74. https://doi.org/10.5271/sjweh.3478

Umanodan, R., Shimazu, A., Minami, M., \& Kawakami, N. (2014). Effects of Computerbased Stress Management Training on Psychological Well-being and Work Performance in 


\section{Macrothink}

International Journal of Learning and Development

ISSN 2164-4063 2018, Vol. 8, No. 3

Japanese Employees: A Cluster Randomized Controlled Trial. Industrial health, 52(6), 480-491. https://doi.org/10.2486/indhealth.2013-0209

Van Straten, A., Cuijpers, P., \& Smits, N. (2008). Effectiveness of a web-based self-help intervention for symptoms of depression, anxiety, and stress: randomized controlled trial. Journal of medical Internet research, 10(1), e7. https://doi.org/10.2196/jmir.954

Van Wingerden, J., Bakker, A. B., \& Derks, D. (2017). Fostering employee well-being via a job crafting intervention. Journal of Vocational Behavior, 100, 164-174. https://doi.org/10.2196/jmir.954

Villani, D., Grassi, A., Cognetta, C., Toniolo, D., Cipresso, P., \& Riva, G. (2013). Self-help stress management training through mobile phones: An experience with oncology nurses. Psychological Services, 10(3), 315. https://doi.org/10.1037/a0026459

Wolever, R. Q., Bobinet, K. J., McCabe, K., Mackenzie, E. R., Fekete, E., Kusnick, C. A., \& Baime, M. (2012). Effective and viable mind-body stress reduction in the workplace: A randomized controlled trial. Journal Of Occupational Health Psychology, 17, 246-258. https://doi.org/10.1037/a0027278

Yamagishi, M., Kobayashi, T., Kobayashi, T., Nagami, M., Shimazu, A., \& Kageyama, T. (2007). Effect of web - based assertion training for stress management of Japanese nurses. Journal of nursing management, 15(6), 603-607. https://doi.org/10.1111/j.1365-2834.2007.00739.x

Yamagishi, M., Kobayashi, T., \& Nakamura, Y. (2008). Effects of web-based career identity training for stress management among Japanese nurses: a randomized control trial. Journal of occupational health, 50(2), 191-193. https://doi.org/10.1539/joh.L7086

Zetterqvist, K., Maanmies, J., Ström, L., \& Andersson, G. (2003). Randomized controlled trial of internet-based stress management. Cognitive Behaviour Therapy, 32(3), 151-160. https://doi.org/10.1080/16506070302316

\section{Copyright Disclaimer}

Copyright for this article is retained by the author(s), with first publication rights granted to the journal.

This is an open-access article distributed under the terms and conditions of the Creative Commons Attribution license (http://creativecommons.org/licenses/by/4.0/). 